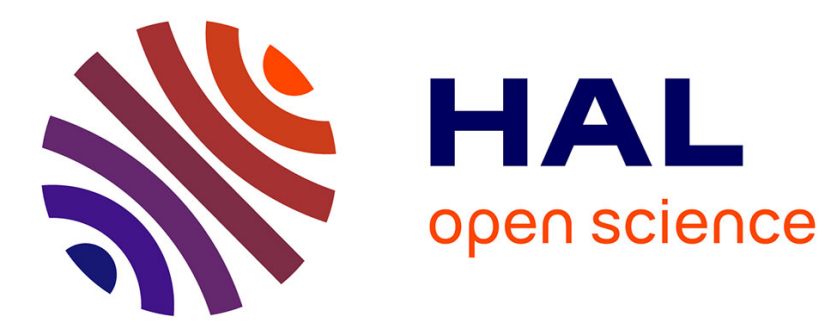

\title{
Experimental investigation of intracavity Bragg gratings
}

Ludivine Menez, Isabelle Zaquine, Alain Maruani, Robert Frey

\section{To cite this version:}

Ludivine Menez, Isabelle Zaquine, Alain Maruani, Robert Frey. Experimental investigation of intracavity Bragg gratings. Optics Letters, 2002, 27 (7), pp.476-478. hal-00866498

\section{HAL Id: hal-00866498 \\ https://hal-iogs.archives-ouvertes.fr/hal-00866498}

Submitted on 26 Sep 2013

HAL is a multi-disciplinary open access archive for the deposit and dissemination of scientific research documents, whether they are published or not. The documents may come from teaching and research institutions in France or abroad, or from public or private research centers.
L'archive ouverte pluridisciplinaire HAL, est destinée au dépôt et à la diffusion de documents scientifiques de niveau recherche, publiés ou non, émanant des établissements d'enseignement et de recherche français ou étrangers, des laboratoires publics ou privés. 


\title{
Experimental investigation of intracavity Bragg gratings
}

\author{
L. Menez, I. Zaquine, and A. Maruani \\ Ecole Nationale Supérieure de Télécommunications, Département Traitement du Signal et de l'Image, \\ 46 Rue Barrault, 75634 Paris Cedex 13, France \\ R. Frey \\ Ecole Nationale Supérieure de Télécommunications, Département Traitement \\ du Signal et de l'Image, 46 Rue Barrault, 75634 Paris Cedex 13, France, and \\ Laboratoire Charles Fabry de l'Institut d'Optique, Institut d'Optique Théorique et Appliquée and \\ Centre National de la Recherche Scientifique, Batiment 503, BP 147, 91403 Orsay Cedex, France
}

Received October 8, 2001

\begin{abstract}
We report the results of an experimental investigation of the diffractive properties of a thick index grating embedded in a Fabry-Perot resonator, the so-called intracavity Bragg grating. We compare the performance of this device with that of a bare Bragg grating with same thickness and the same index modulation and establish the improvement in performance with the resonator. The experimental data also fit predictions calculated from a theoretical model. () 2002 Optical Society of America

OCIS codes: $090.1970,090.7330,190.4360$.
\end{abstract}

Real-time holography is a fast and powerful tool for data processing. It requires high diffraction efficiencies, obtained with thick samples or materials with significant refractive-index modulation. In all cases, the diffraction performance of a given nonlinear medium can be enhanced by a resonator, which increases the optical path. Theoretical and experimental investigations have already proved the advantage of using a Fabry-Perot etalon with quantum wells $\mathrm{s}^{1,2}$ or index gratings recorded in photorefractive bulk samples. ${ }^{3,4}$ Recent calculations have shown that the diffraction properties of transmission Bragg gratings are greatly improved when the indexmodulated medium is embedded in a Fabry-Perot resonator. ${ }^{5}$ Specifically, intracavity Bragg gratings can give very high diffraction efficiencies and angular selectivities, even in the case of thin materials or low index-modulation amplitude, which is crucial in optical signal processing. When the Bragg condition and Fabry-Perot resonance are simultaneously fulfilled, the performance of the intracavity device is greatly enhanced, in comparison with that of a bare sample.

In this Letter we present an experimental investigation of intracavity Bragg diffraction in the case of an asymmetrical cavity operated in reflection. This configuration was predicted to give the best results ${ }^{5}$ with respect to the discussion presented above.

The experimental setup is shown in Fig. 1. The intracavity holographic device was composed of an organic dye, MOED ${ }^{6,7}$ dissolved in ethanol $(n=1.3611)$ and enclosed between two dielectric mirrors. This dye was strongly absorbent at short wavelengths (below $600 \mathrm{~nm}$ ) and almost totally transparent at longer wavelengths $(650-850 \mathrm{~nm})$. Two coherent beams of equal intensity, emitted by a frequencydoubled $Q$-switched Nd:YAG laser $\left(\lambda_{W}=532 \mathrm{~nm}\right)$, wrote a thermal-index modulation in the intracavity medium through the absorption of their interference pattern. In this way, the liquid temperature was spatially modulated, inducing a periodic change in the refractive index $\left(\mathrm{d} n / \mathrm{d} T=-3.6 \times 10^{-4} \mathrm{~K}^{-1}\right.$ in ethanol). The period $\Lambda$ of the grating was determined by the refraction angle $\theta_{W}$ of the write beams $\left[\Lambda=\lambda_{W} / 2 n \sin \left(\theta_{W}\right) \approx 4.7 \mu \mathrm{m}\right]$. The fringes, which filled the whole volume of the Fabry-Perot cavity, were oriented perpendicularly to the resonator mirrors, since the two write beams were symmetrical with respect to the cavity axis (Fig. 1). The Nd:YAG laser was externally triggered by a pulse generator so that one pulse was emitted every $4 \mathrm{~s}$. This very low rate was necessary to ensure the total dissipation

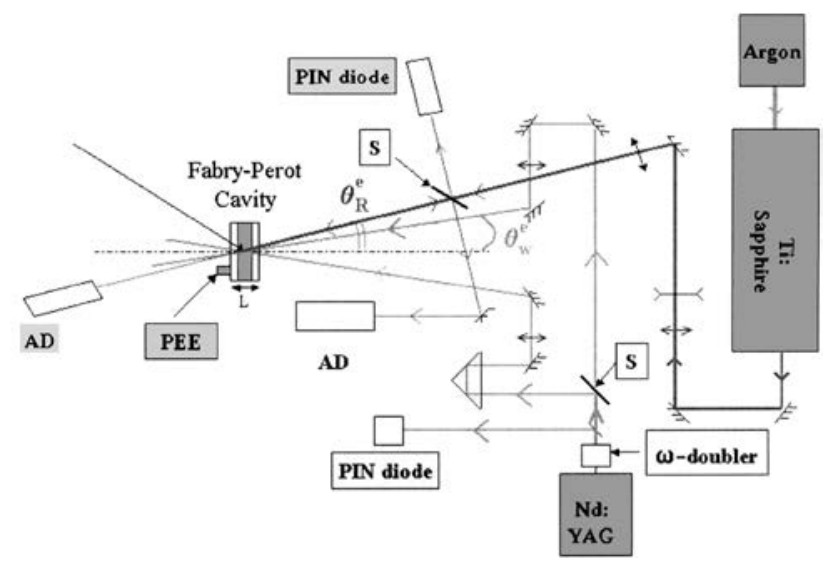

Fig. 1. Sketch of the experimental setup: The write beams are emitted by a pulsed $\omega$-doubled Nd:YAG laser. They converge symmetrically on the Fabry-Perot cavity at external incidence $\theta_{W}{ }^{e}$. The read beam (dark solid line) is emitted by a cw Ti:sapphire laser pumped by an argon laser. The beam's external incidence angle on the device is $\theta_{R}{ }^{e}$. The length of the Fabry-Perot cavity is tuned by a piezo-electric element (PEE). Avalanche detectors (ADs) detect the transmitted and reflected diffracted beams; the latter is extracted by beam splitters (S's); references of the read and write energies are measured with $\mathrm{p}-\mathrm{i}-\mathrm{n}$ photodiodes. 
of heat between two pulses. The pulse duration was $\sim 10 \mathrm{~ns}$, which is much shorter than the thermal diffusion time of the recorded grating $(\sim 6 \mu \mathrm{s}$ for a $4.7-\mu \mathrm{m}$ fringe space), thus giving highly contrasted index modulation. The read beam $\left(\lambda_{R}=780 \mathrm{~nm}\right)$ was produced by a tunable Ti:sapphire laser pumped by a cw argon laser. The refraction angle $\left(\theta_{R}\right)$ of this beam could be tuned by translation of the beam transversely to the axis of the focusing lens. The thickness of the recorded grating was equal to the length of the cavity, $l=730 \mu \mathrm{m}$. The asymmetrical resonator was composed of a partially reflecting front mirror $\left(R_{1} \approx 0.79\right.$ at $\left.\lambda_{R}=780 \mathrm{~nm}\right)$ and an almost totally reflecting back mirror $\left(R_{2} \approx 0.99\right.$ at $\left.780 \mathrm{~nm}\right)$. This kind of cavity is also called a Gires-Tournois interferometer. $^{8}$ A piezoelectric element was sealed on the rear mirror so that the thickness of the cavity could be finely adjusted to Fabry-Perot resonances at fixed $\theta_{R}$ [2nl $\cos \left(\theta_{R}\right)=m \lambda_{R}, m$ being an integer]. A beam splitter reflected a fraction of the incident read beam onto a $\mathrm{p}-\mathrm{i}-\mathrm{n}$ photodiode (the read reference intensity) and a fraction of the reflected diffracted beam was sent to a fast avalanche photodiode, which allowed measurement of the instantaneous diffraction efficiency in reflection, $\rho_{\mathrm{DR}}$.

To optimize the efficiency of the intracavity device, we first looked for the value of refraction angle of the read beam, $\theta_{R}$, that fulfilled the Bragg condition: For each $\theta_{R}$, we optimized the diffraction efficiency $\left(\rho_{\mathrm{DR}}\right)$ by tuning the thickness of the Fabry-Perot cavity. The optimal diffraction efficiency $\left(\rho_{\text {DRopt }}\right)$ was obtained for $\theta_{R}=\theta_{\text {Bragg }}=a \sin \left(\lambda_{R} / 2 n \Lambda\right)$. Then we investigated the influence of detuning from either the Fabry-Perot resonance or the Bragg condition. For $\theta_{R}=\theta_{\text {Bragg, }}$, with a length change $\Delta l$ from Fabry-Perot resonance $\left[2 n l \cos \left(\theta_{\text {Bragg }}\right)=m \lambda_{R}\right]$, we measured the variations of $\rho_{\mathrm{DR}}$ with the phase shift, $\Phi_{\mathrm{FP}}=4 \pi n \Delta l \cos \left(\theta_{R}\right) / \lambda_{R}$. To quantify $\Phi_{\mathrm{FP}}$, we used the measurement of the intensity transmitted by the cavity with the written beams turned off, when a $50-\mathrm{Hz}$ sinusoidal high voltage was applied to the piezoelectric element. Through the observation of two consecutive resonance peaks of the Fabry-Perot cavity we could establish a calibration of the phase mismatch, $\Phi_{\mathrm{FP}}$, as a function of the measured transmitted intensity, assuming that the piezoelectric element was operated in a linear regime.

When the write Nd:YAG laser was turned on, the phase mismatch was tuned by application of a cw high voltage to the piezoelectric element. For each pulse, the references of the incident write and read beams and those of the read transmitted and reflected diffracted beams were measured with photodiodes and avalanche detectors. Figure 2 shows the experimental reflection diffraction efficiency $\left(\rho_{\mathrm{DR}} \exp \right)$ and its calculated value $\left(\rho_{\text {DR }}\right.$ calc) versus the Fabry-Perot phase mismatch, $\Phi_{\mathrm{FP}}$, measured at $\theta_{R}=\theta_{\mathrm{Bragg}}$. The shape of the reflection diffraction peak was described mainly by the Fabry-Perot transmission, since only light coupled to the cavity could be diffracted by the Bragg grating. As expected from plane-wave calculations, ${ }^{5}$ the angular width of $\rho_{\mathrm{DR}}$ was strongly reduced (by roughly a factor of 7) compared with the corresponding theoretical results for the bare sample. Moreover, the absolute maximum diffraction efficiency was $\sim 4 \times 10^{-2}$, i.e., 220 times higher than that of the bare Bragg sample with the same thickness and index modulation. We obtained the fit shown in Fig. 2 (solid curve) by taking the Gaussian shape of the read beam and the small absorption of the intracavity medium into account. Indeed, because of the limited width (beam radius, $0.3 \mathrm{~mm}$ ) of the oblique read beam, the observed finesse $\left(\mathcal{F}_{\text {obs }}=9\right)$ of the Fabry-Perot cavity was much smaller than the theoretical value $\left(\mathcal{F}_{\text {th }}=25\right)$. The curve shown in Fig. 2 was obtained for $1.4 \%$ intracavity losses and with refractive-index modulation $\Delta n=4.7 \times 10^{-6}$, in close agreement with the value $\Delta n=4.6 \times 10^{-6}$ ' calculated from the absorbed energy of the write beams by use of the thermal properties of ethanol.

We also studied the variations of the diffraction efficiency of a resonant cavity with detuning from Bragg condition by tuning the incidence angle $\theta_{R}$ of the read beam. Because the calculated FWHM of the intracavity angular resonant peak was $\sim 0.4 \mathrm{mrad}$, the maximum reflection diffraction efficiency was measured for incidence angles regularly spaced between $\theta_{\text {Bragg }}-\delta \theta$ and $\theta_{\text {Bragg }}+\delta \theta$, with $\delta \theta=1.25 \mathrm{mrad}$. For each $\theta_{R}$, we tuned $\Phi_{\mathrm{FP}}$ and looked for the maximum value of the Fabry-Perot transmission. In Fig. 3 we present the normalized results and the intracavity $\rho_{\mathrm{DR}}$ calculated for a resonant Fabry-Perot cavity with the same values for the parameters as in the case of Fig. 2. These two quantities are plotted versus the angle variation, $\Delta \theta=\theta_{R}-\theta_{\text {Bragg. }}$. These two plots are in good agreement and exhibit a FWHM of $0.6 \mathrm{mrad}$, which is roughly seven times smaller than the value calculated for the bare sample $\left(\mathrm{FWHM}_{\text {bare }} \approx 4.3 \mathrm{mrad}\right)$. This result confirms the improvement obtained with

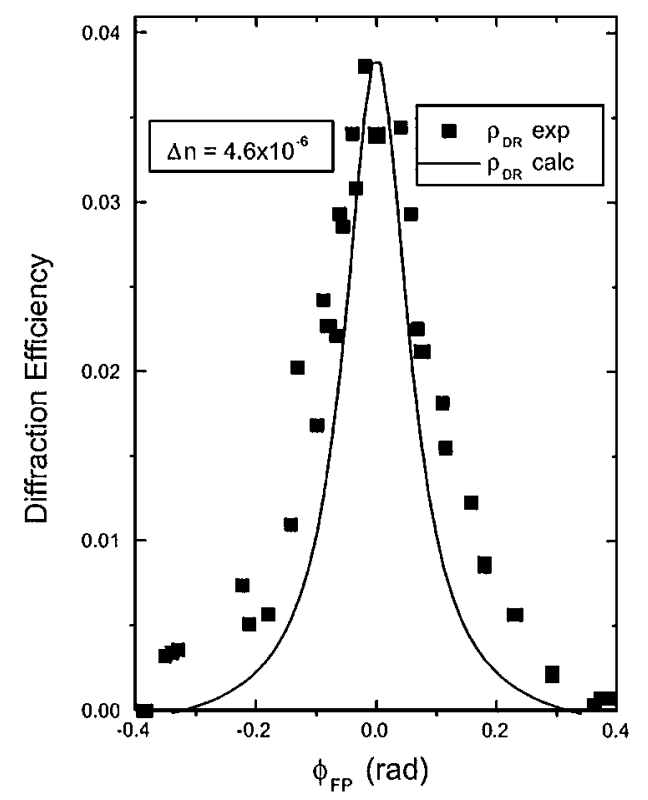

Fig. 2. Experimental data and numerical calculations of the diffraction efficiency, $\rho_{\mathrm{DR}}$, versus the Fabry-Perot phase mismatch. The cavity length is $l=730 \mu \mathrm{m}$, the grating period is $\Lambda \approx 4.7 \mu \mathrm{m}$, and the read wavelength is $\lambda_{R}=780 \mathrm{~nm}$. 


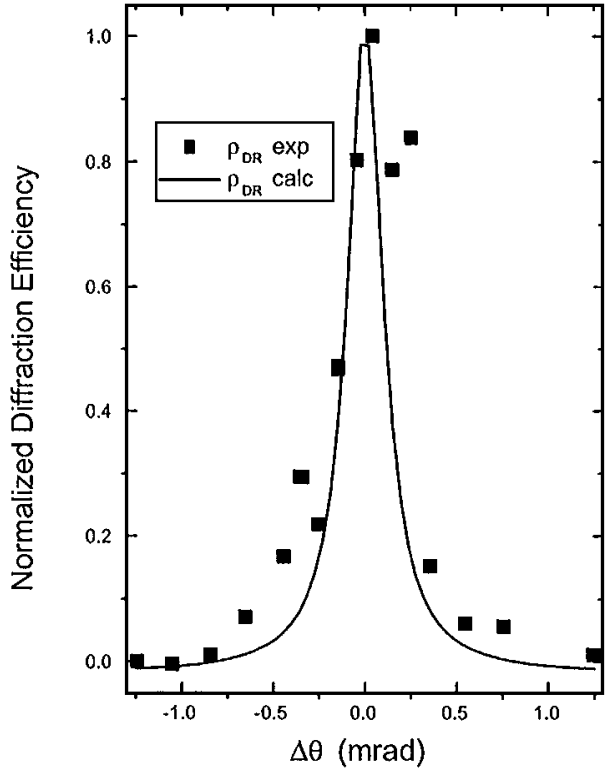

Fig. 3. Experimental data and numerical calculations of the diffraction efficiency, $\rho_{\mathrm{DR}}$, versus the angular detuning $\Delta \theta$ from Bragg resonance.

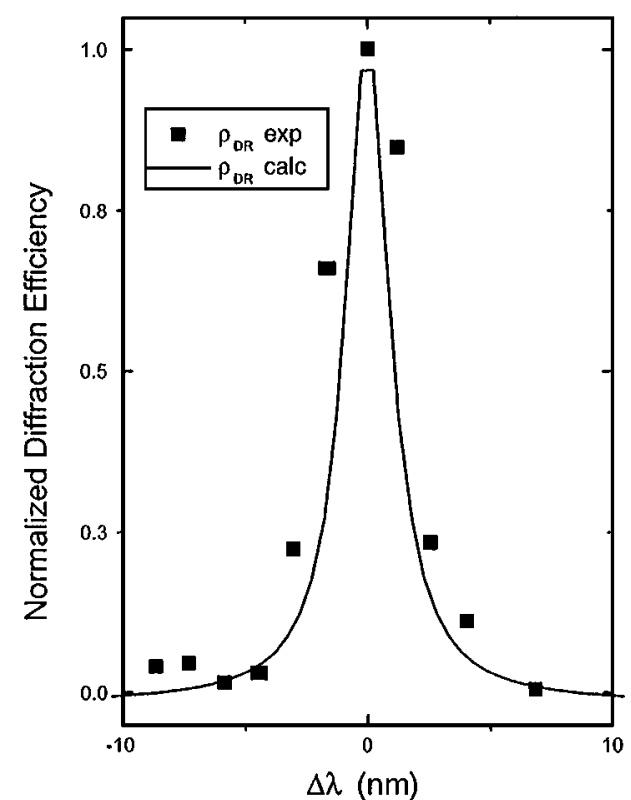

Fig. 4. Experimental data and numerical calculations of the diffraction efficiency, $\rho_{\mathrm{DR}}$, versus the spectral detuning $\Delta \lambda$ from Bragg resonance.

the resonator. In addition, for small values of $\Delta \theta$ we could measure higher diffraction efficiencies with a slightly detuned cavity: The Fabry-Perot and the Bragg mismatches partly compensate for each other in this case.

We also investigated the diffraction efficiency with incidence angles much different from $\theta_{\mathrm{Bragg}}$, particu- larly around the supposed side resonances of the bare Bragg grating. We could not measure any significant diffraction efficiency for those incidence angles: The detected signal could not be extracted from the surrounding noise. Nevertheless, an overestimation of the relative intensity of those side resonances was $1 \%$ of the maximum diffracted intensity. The signalto-noise ratio is then more than five times larger than that of the bare sample.

The Bragg resonance was also investigated by tuning of the wavelength of the Ti:sapphire read beam $\left(\lambda_{R}\right)$. For each $\lambda_{R}$, we measured the $\rho_{\mathrm{DR}}$ at Fabry-Perot resonance and explored the $[775 \mathrm{~nm}$; $810 \mathrm{~nm}]$ bandwidth. The experimental and the calculated spectral variations of normalized $\rho_{\mathrm{DR}}$ for a resonant cavity are plotted in Fig. 4 . The theoretical predictions matched the measurements for the same values of the parameters as in the previous experiments. The intracavity device exhibits a spectral width at half-maximum of $\Delta \lambda \approx 10 \mathrm{~nm}$, whereas the calculated value for the equivalent bare transmission Bragg grating is $\Delta \lambda \approx 40 \mathrm{~nm}$. The spectral selectivity of the Bragg grating is enhanced by the resonator but remains much lower than that obtained with reflection Bragg gratings.

In conclusion, significant improvements in maximum diffraction efficiency ( 2 orders of magnitude) and angular selectivity (7 times greater) have been experimentally demonstrated when Bragg gratings were placed inside a Gires-Tournois interferometer. The spectral selectivity and the signal-to-noise ratio were also enhanced, in a perhaps less spectacular but nonnegligible way. With an optimized device, such an intracavity Bragg grating could be used for applications requiring high selectivity and a high signal-to-noise ratio, such as multiplexers or filters, especially if low-thickness samples must be used.

The authors thank Pr. Delaire of the Ecole Normale Supérieure de Cachan for providing the MOED sample. I. Zaquine's e-mail address is isabelle. zaquine@enst.fr.

\section{References}

1. D. D. Nolte and K. M. Kwolek, Opt. Commun. 115, 606 (1995).

2. K. M. Kwolek, M. R. Melloch, and D. D. Nolte, Appl. Phys. Lett. 67, 736 (1995).

3. Q. Byron He, P. Yeh, and C. Gu, Opt. Lett. 17, 664 (1992).

4. Q. B. He, H. K. Liu, and P. Yeh, Appl. Phys. B 59, 467 (1994).

5. L. Menez, I. Zaquine, A. Maruani, and R. Frey, J. Opt. Soc. Am. B 16, 1849 (1999).

6. M. J. Minch and S. S. Shah, J. Chem. Educ. 54, 709 (1977).

7. A. P. Phillips, J. Org. Chem. 14, 302 (1949).

8. F. Gires and P. Tournois, C. R. Acad. Sci. 258, 6112 (1964). 